\title{
Functional Rehabilitation of Mandibular Molar with Separated Instrument and External Root Resorption
}

\author{
${ }^{1}$ Sachin Shashikant Metkari, ${ }^{2}$ Praneeta S Kamble, ${ }^{3}$ Rajesh Kumar Meena, ${ }^{4}$ Manoj Mahadeo Ramugade
}

\begin{abstract}
Procedural errors like separated instrument have always been a hindrance for successful endodontic therapy. However, it becomes more challenging when there is an added condition of root resorption in the same root in which instrument is separated. Here, we present and discuss such a case where instrument retrieval was unsuccessful and had to restore to surgical techniques like hemisection and extraction of affected root of the tooth followed by prosthetic rehabilitation.
\end{abstract}

Keywords: Separated instrument, Root resorption, Hemisection.

How to cite this article: Metkari SS, Kamble PS, Meena RK, Ramugade MM. Functional Rehabilitation of Mandibular Molar with Separated Instrument and External Root Resorption. Int J Prosthodont Restor Dent 2015;5(1):27-30.

\section{Source of support: Nil}

Conflict of interest: None

\section{INTRODUCTION}

Endodontic mishap like separation of instruments in the root canal is always a challenge for successful endodontic treatment. Even if we manage to retrieve the separated instrument, sometimes future fracture of tooth is impediment in successful endodontic therapy. ${ }^{1}$ But, root resorption with separated instrument in the same root will certainly have hard time for clinician to save the tooth.

Here in our case, we manage to save such a molar tooth by hemisection and amputation of affected root followed by prosthetic rehabilitation with 2 years followup evaluation.

\section{CASE REPORT}

A 22 years old female patient with noncontributory medical history reported in the department of conservative

\footnotetext{
${ }^{1,4}$ Assistant Professor, ${ }^{2}$ Additional Professor

${ }^{3}$ Postgraduate Student

${ }^{1,4}$ Department of Conservative Dentistry and Endodontics, Nair Hospital Dental College, Mumbai, Maharashtra, India

${ }^{2,3}$ Department of Periodontics, Nair Hospital Dental College Mumbai, Maharashtra, India
}

Corresponding Author: Sachin Shashikant Metkari, Assistant Professor, Department of Conservative Dentistry and Endodontics, Nair Hospital Dental College, Mumbai, Maharashtra India, Phone: 02223082714, e-mail: drssmetkari@yahoo.co.in dentistry and endodontics with complain of pain in lower left posterior region of the mouth since 1 to 2 months. Pain was dull, intermittent and of short duration which aggravated on cold stimulation. Patient gave history of root canal treatment of \#36 (FDI numbering system) 8 months back. On examination, tooth \#36 was very sensitive to vertical and horizontal percussion. Affected lower left posterior region had normal periodontal support (Figs 1 and 2).

Intraoral periapical radiograph of the involved tooth \#36 showed deficient obturation and possibly separated instrument at the middle-apical third of mesial root of \#36 about 3 to $4 \mathrm{~mm}$ in length. Periapical radiolucency about 2 to $3 \mathrm{~mm}$ in diameter was observed around mesial root. There was root as well as bone resorption evident on radiograph in apical portion of mesial root. Cone beam

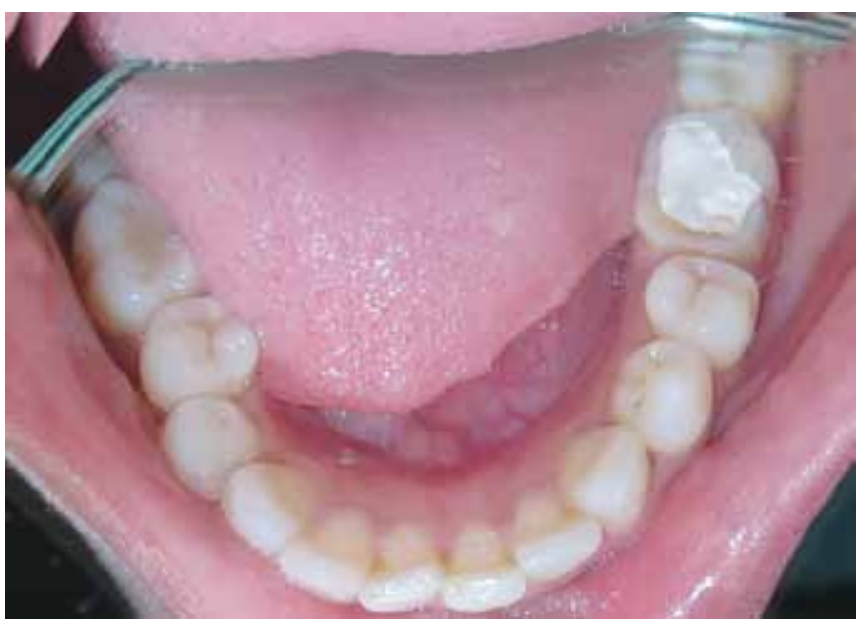

Fig. 1: Preoperative view showed temporary restoration in \#36

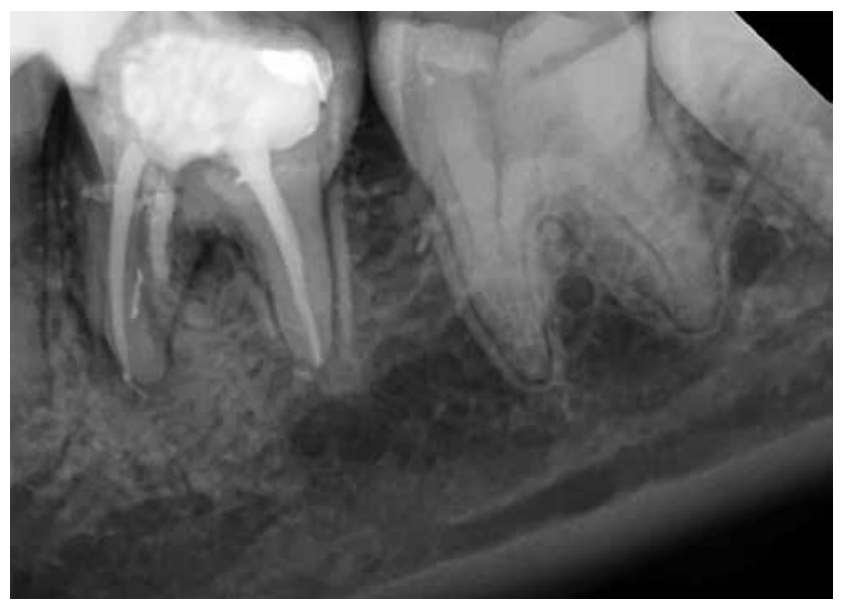

Fig. 2: Intraoral periapical radiograph of \#36 
computed tomography (CBCT) confirmed the presence of separated instrument in mesiolingual canal at 3 to $4 \mathrm{~mm}$ short of the apex of \#36.

\section{TREATMENT PLAN}

It was decided to retreat the tooth by nonsurgical technique to correct the inadequate obturation and remove the separated instrument from \#36 followed by treatment of periapical lesion. If instrument has not been retrieved successfully then hemisection and extraction of affected half of tooth would be planned. ${ }^{2}$ All these treatment modalities along with prognosis were explained to the patient, and informed written consent was obtained.

\section{PROCEDURE}

Instrument retrieval was attempted with Masserann's kit under surgical operating microscope. Maximum attempts did not yield any results. Further attempts would have caused thinning of mesial root and/or strip perforation of affected root, therefore, second line of treatment, i.e. hemisection along with extraction of mesial root was considered..$^{3-5}$

\section{HEMISECTION AND ROOT AMPUTATION}

Inferior alveolar nerve block anesthesia (2\% lignocaine with epinephrine in 1:80000) was administered and full thickness periodontal flap was raised with crevicular incision from first premolar (\#34) to second molar (\#37). Buccal and lingual periodontal flaps were reflected. A long shank tapered fissure carbide bur (169L) was used to make vertical cut towards the furcation area to bisect mesial and distal halves of \#36. A fine probe was passed through the cut to ensure separation ${ }^{4-8}$ (Figs 3 to 5).

The mesial half of \#36 was extracted and remaining distal half was smoothened. The surgical field area was

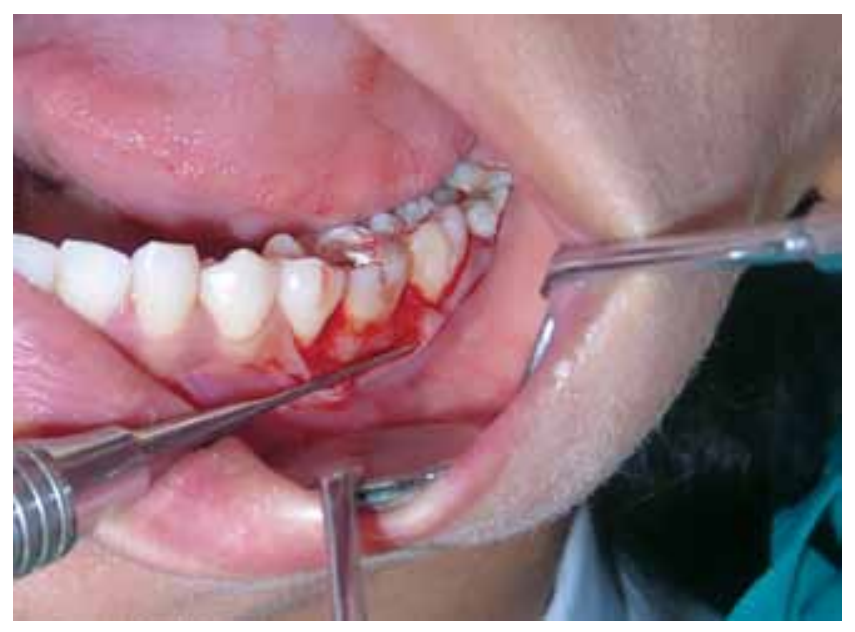

Fig. 3: Full thickness periodontal flap raised



Fig. 4: Separated mesial and distal halves of \#36

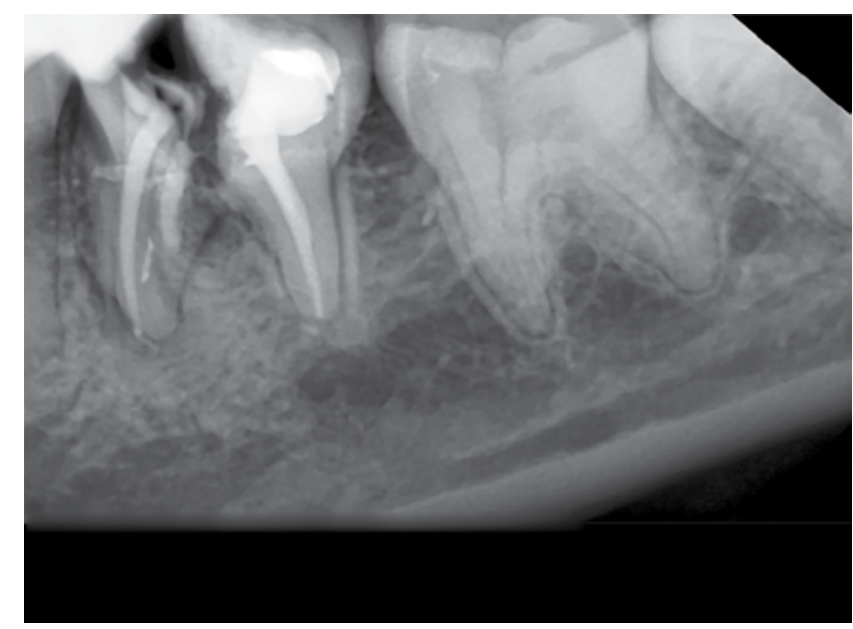

Fig. 5: Intraoral periapical radiograph showing separated mesial and distal halves of \#36

flushed with saline and chlorhexidine (0.02\%) to ensure no residual debris (Figs 6 and 7). Mesial root socket was filled with bone graft (demineralized freezed dried bone allograft DFDBA) and suturing (black braided silk suture 3-0) was done followed by Coe-Pak application. Patient was recalled for check-up after 24 hours. Periodontal pack and sutures were removed after 10 days.

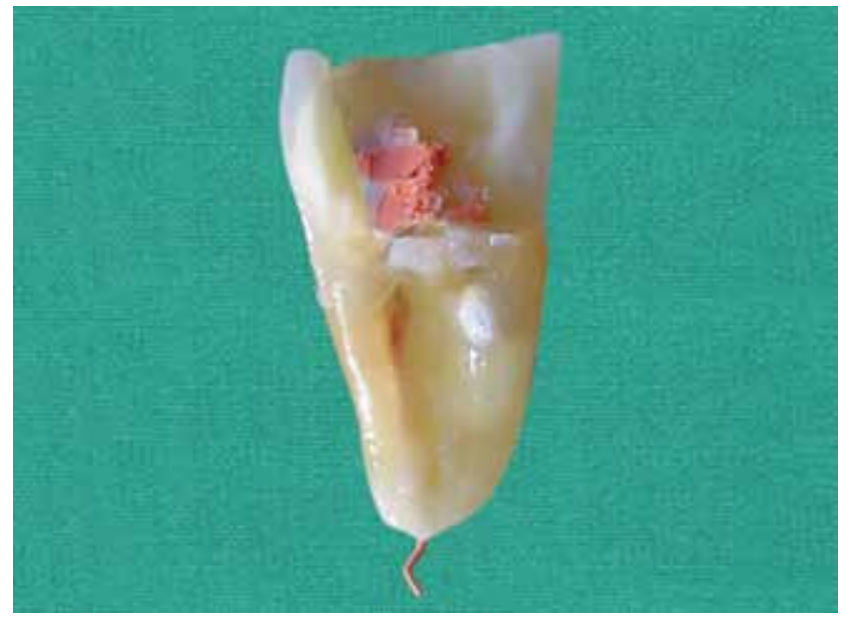

Fig. 6: Extracted mesial half of \#36 with apical resorption 


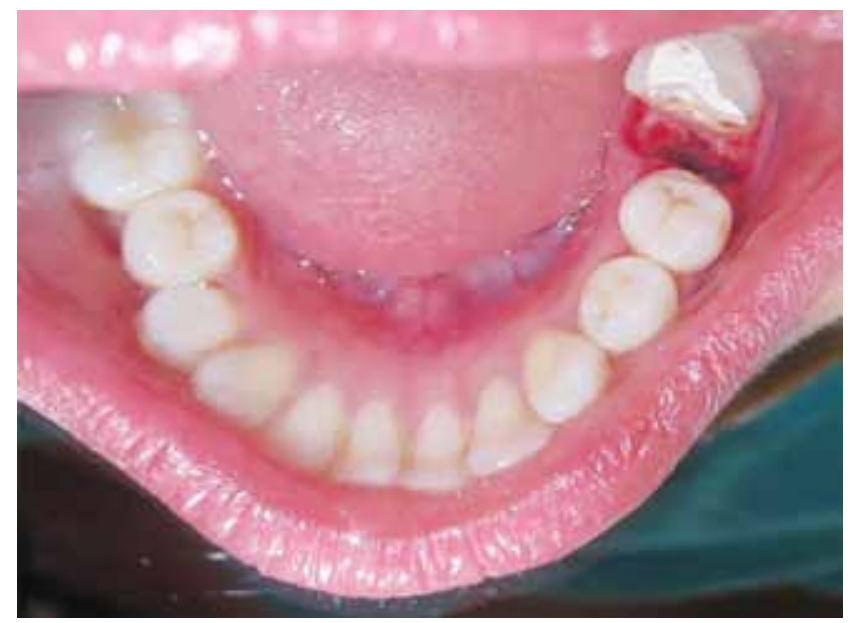

Fig. 7: Intraoral view showing extraction socket of mesial half of \#36

For the prosthetic rehabilitation, temporary partial prosthesis was placed in \#35 and \#36 and kept under observation for 2 months. Later on, patient reported with severe sensitivity to hot and cold beverages in relation to \#35. So, endodontic therapy was done with \#35, and fixed partial denture was made with \#35 and \#36 (Fig. 8).

Patient was recalled for periodic evaluation after 1 to 3 months and 1 to 2 years.

\section{DISCUSSION}

In reported case, we have focused on an alternative treatment option for salvaging the healthy tooth structure when patient desired to save natural tooth rather than succumbing tooth to extraction. In this case, the tooth had a very healthy periodontium supporting the distal root of \#36. The root also had sound straight root structure to act as a strong support for crown and bridge prosthesis.

Removal or bypass of separated instrument is necessary for successful cleaning and shaping of root canal system. ${ }^{4}$ Easy removal of the separated instrument depends upon the anatomy of the tooth which includes

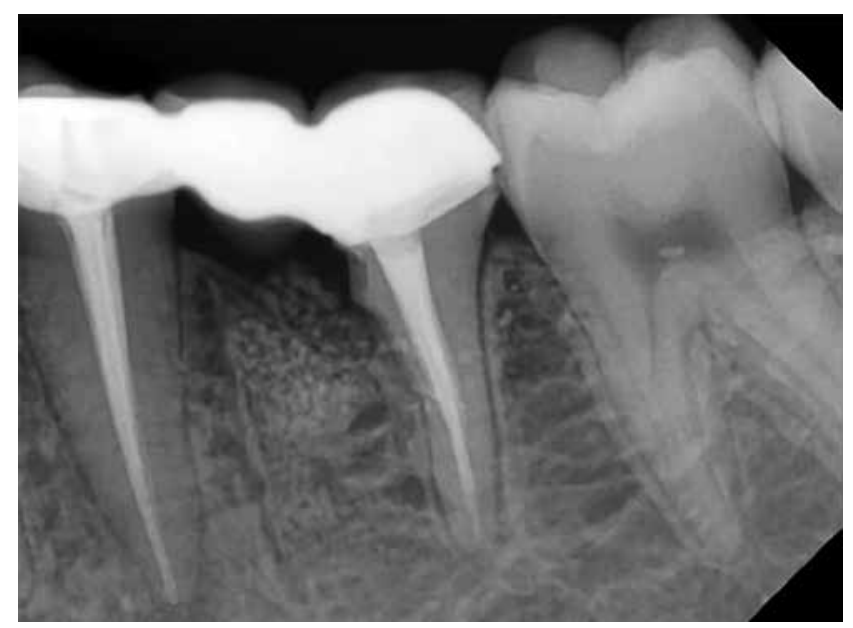

Fig. 8: Prosthetic rehabilitation of \#35 and \#36 the length, diameter, canal curvature and additionally limited by root morphology which includes the thickness of dentin and the depth of external concavities of root. ${ }^{4}$ Majority of the times, separated instrument retrieval procedure leads to weakening of tooth structure which may cause fracture and, thus, loss of the tooth, all this is due to overzealous canal enlargement. ${ }^{3,4}$

Many times, instruments are separated apical to the curvature of the canal. In such cases, a safe access to the site of separation may not be achieved. The removal of the fractured instrument is not possible, so surgery or extraction of a tooth will be needed at times in presence of adverse signs and symptoms. Here in this case, we observed root as well as bone resorption at the apical portion of root.

Considering all these factors, we planned for hemisection and amputation of mesial part of a tooth as a less detrimental approach than extraction of whole tooth. Hemisection is the splitting of a two-rooted tooth into two separate portions. The term root amputation and hemisection are collectively known as root resection. ${ }^{6-8}$ In root resection procedure, we retain most of the tooth structure but the disadvantage is that remaining root or roots must undergo endodontic therapy and crown must be placed as restorative management. ${ }^{6,7}$ Hence in our case, we resect the affected mesial half followed by root canal therapy and rehabilitate the remaining segment using fixed partial denture with \#35 and \#36.

\section{CONCLUSION}

Prevention is the best strategy and using safe techniques during root canal preparation procedures may virtually eliminate the accidental fracture of instrument during procedure. Separation of the instrument can be prevented if the instruments used for negotiating and cleaning and shaping the root canal are disposed after use for that particular tooth.

Discarding all instruments after the completion of each endodontic case will reduce breakage, save clinical time and upsets caused by procedural accidents.

However, on occasion an instrument might break and in spite of the best existing technologies and techniques the retrieval may not be successful. In these instances according to situation, root resection is advised and prosthetic rehabilitation to be done.

\section{ACKNOWLEDGMENT}

We would like to thank to Professor Dr KS Banga and Associate Professor Dr Kishor Sapkale, for guiding and encouragement. 


\section{REFERENCES}

1. Parashos $\mathrm{P}, \mathrm{Messer} \mathrm{HH}$. Rotary Ni-Ti instrument fracture and its consequences. J Endod 2006;32(11):1031-1043.

2. Buhler HH. Survival rates of hemisected teeth: an attempt to compare them with the survival rates of alloplatic implant. Int J Periodon Res Dent 1994;14(6):536-543.

3. Ming-Gene Tu, San-Yue Chen, Shui-Shang Hsue, Heng-Li Huang, Chi-Cheng Tsai. Removal of a separated nickeltitanium instrument from a three rooted mandibular first molar. Mid Taiwan J Med 2009;14(1):27-33.

4. Shenoy, Mandava, Bolh, Vemuri. A novel technique for removal of broken instrument from root canal in mandibular second molar. Ind. J Dent Res 2014;25(1): 107-110.

5. Pai AR, Kamath MP, Basnet P. Retrival of separated file using Masserann Technique: a case report. Kathmandu Univ Med J 2006;4(2):238-242.

6. Abrams LL. Hemisection-technique and restoration. Dental Clinics of North America 1974;18(24):15-44.

7. Kost WJ. Root amputation and hemisection. J Canad Dent Asso 1991;57(1):42-45.

8. Shah S, Modi B, Desai K, Duseja SD. Hemisection: a conservative approach for a periodontally compromised tooth—a case report. J Adv Oral Research 2012;3(2):31-35. 